\title{
The Cartan algebra of exterior differential forms \\ as a supermanifold: morphisms and manifolds associated with them
}

\author{
ARMIN UHLMANN
}

Karl-Mard-University Leipzig Department of Physics and NTZ

\begin{abstract}
A$ supermanifold, $M^{m / n}$, can be caracterired by its smooth superfunctions which constitute an algebra A (Leites, Kostant). We associate canonically ka la Gelfand , certain fibred manifolds on which the automorphisms (the Jordan automorphisms) of A act as diffeomorphisms. For example, the kernels of all homomorphisms from the algebra of superfunctions onto the Grassmann algebra of dimension $n$ form naturally a manifold of dimension $m 2^{n-1}$ if $n$ is even. To be more specific we explain this and similar constructions in the case of the algebra of smooth exterior differential forms defined on a smooth manifold. This algebra defines a particular supermanifold $M^{m / m}$.
\end{abstract}

\section{INTRODUCTION}

A smooth supermanifold $M^{m / n}$ (see [1, 2, 3, 4]) uniquely defines the algebra of smooth superfunctions. Locally, superfunctions can be considered as germs of (smooth) maps of a smooth manifold into the unital Grassmann algebra with $n$ generators over the real or complex numbers. We consider here the real case. It is known that in the smooth, i.e. class $C^{\infty}$ case the algebra of superfunctions

Key-Words: Supergeometry, Exterior Differential Forms, Associative Algebra, Automorphisms, Jordan Automorphisms.

1980 Matbematics Subject Classification: 58 A 15, 16 A 68,57 R 50.

This article is based on lectures given by the Author during the Trimester on Mathematical Physics at the Stefan Banach International Mathematical Centre, Warsaw, Sept. - Nov. 1983. 
completely characterizes the supermanifold.

Let $\mathrm{A}$ be the algebra of real and smooth superfunctions defined on $M^{\mathrm{m} / \mathrm{n}}$. Because $\mathbf{A}$ is an algebra with a large radical its maximal ideals are too coarse to give more than the structure of the base manifold $M$. This changes, however, in considering other classes of ideals and related structures.

Thus the set «G-space $M^{m / n} »$ of all ideals, $\mathbf{J}$, of $\mathbf{A}$ with the property that the factor algebra $\mathbf{A} / \mathbf{J}$ is isomorphic to the unital real Grasmann algebra with $n$ generators naturally carries the structure of a smooth manifold: Indeed, there is only one way to equip this set of ideals with the structure of a smooth manifold allowing the representation of all automorphisms of $\mathbf{A}$ as diffeomorphisms. The dimension of this manifold equals

$$
m 2^{n-1} \quad \text { resp. } \quad m\left(2^{n-1}+1\right)
$$

depending wether $n$ is even or odd.

Likewise, the set $« J G$-space $M^{m / n}$ » consisting of all Jordan ideals of A with A/J Jordan isomorphic to the real unital Grassmann algebra of $n$ generators may be considered a manifold on which all Jordan automorphisms of our algebra of superfunctions act as diffeomorphism.

Evidently one can construct in the manner indicated above even higher dimensional spaces by considering not the set of kernels but the set of (Jordan) homomorphisms of A onto the Grassmann algebra. To control this behaviour one needs to know a large enough set of automorphisms and Jordan automorphisms of the algebra of superfunctions. It is, indeed, possible to enumerate all of them $[5,6]$ but we shall restrict ourselves to those we need.

Further, we shall not go into the most general smooth supermanifold though this would be possible with the methods explained below. To be more definite we will restrict ourselves (with the exeption of the next section) to the Cartan algebra of smooth real exterior differentials on a smooth manifold $M$. Then $m=n$.

A final remark is the following: Starting not with the real but with the complex Grassmann-valued superfunctions we get, by performing the announced construction, quite other spaces. Namely, the set of kernels which form the « $G$-space», the « $J G$-space», and so on is much larger than in the real case, and the groups of (Jordan) automorphisms are of correspondingly higher dimensions.

\section{SUPERCOMMUTATIVE ALGEBRAS}

Let $\mathbf{A}$ be an algebra with unit element which we denote by $1_{A}$ or simply by 1 . For definiteness only we assume $\mathbf{A}$ to be an algebra over the field of real numbers.

A supercommutative algebra is a pair consisting of an algebra $\mathbf{A}$, and an auto- 
morphism, $w$, fulfilling

i) $w^{2}=\mathrm{id}$.

ii) if $w(\mathbf{a})=\mathbf{a}$ then $\mathbf{a}$ is contained in the centre of $\mathbf{A}$

iii) if $w(\mathbf{a})=-\mathbf{a}$ then $\mathbf{a}^{2}=0$.

An element $\mathbf{a} \in \mathbf{A}$ is called «even» if $w(\mathbf{a})=\mathbf{a}$ and «odd» if $w(\mathbf{a})=-\mathbf{a}$. The set of all even resp. odd elements of the algebra we denote by $\mathbf{A}_{+}$respectively $\mathbf{A}_{\ldots}$. There is a direct sum decomposition

$$
\mathbf{A}=\mathbf{A}_{+}+\mathbf{A}_{-} \text {. }
$$

The radical, $\mathbf{S}$, of an algebra $\mathbf{A}$ is by definition the intersection of all its maximal ideals.

PROPOSITION 1. The odd part, $\mathbf{A}_{-}$, of every supercommutative algebra, $\mathbf{A}$, is contained in its radical, $\mathrm{S}$.

$$
\mathbf{A} \subseteq \mathbf{S} \text {. }
$$

Indeed, let $b_{1}, \ldots, b_{k}$ denote odd elements. By using decomposition (1) and the properties of $w$ we see that every element of the form

$$
\mathbf{a}_{1} \mathbf{b}_{1} \mathbf{c}_{1}+\ldots+\mathbf{a}_{k} \mathbf{b}_{k} \mathbf{c}_{k}
$$

is nilpotent for any choice of the $\mathbf{a}_{j}$, $\mathbf{c}_{j}$ out of $\mathbf{A}$. Hence the ideal generated by A consists of nilpotent elements only and is, therefore contained in every maximal ideal.

A Jordan subalgebra, $\mathbf{F}$, of $\mathbf{A}$ is a linear subspace containing $\mathbf{a}^{2}$ if it contains a.

We denote by $P$ the projection operator onto the even part of $\mathbf{A}$.

$$
P \mathbf{a}=(1 / 2)(w(\mathbf{a})+\mathbf{a}), \quad \mathbf{a} \in \mathbf{A} .
$$

PROPOSITION 2. Let $\mathbf{F}$ be a Jordan subalgebra of A. Then

$$
\mathbf{F}_{+}=P(\mathbf{F})
$$

is a subalgebra of $\mathbf{A}$.

Proof. Let $\mathbf{a}, \mathbf{b} \in \mathbf{F}$ and denote by $\mathbf{a}_{+}, \mathbf{b}_{+}$their even parts which are in $\mathbf{F}_{+}$. Then $P(\mathbf{a b}+\mathbf{b a})=\mathbf{a}_{+} \mathbf{b}_{+}+\mathbf{b}_{+} \mathbf{a}_{+}+\mathbf{a}_{-} \mathbf{b}_{-}+\mathbf{b}_{-} \mathbf{a}_{-}$, which equals $2 \mathbf{a}_{+} \mathbf{b}_{+}$by supercommutivity, is in $\mathbf{F}_{+}$.

DEFINITION. A Jordan subalgebra, F, of A is called «splitting» iff 


$$
\mathbf{A}=\mathbf{F}+\mathbf{S}, \quad \mathbf{F} \cap \mathbf{S}=\{0\}
$$

PROPOSITION 3. Let $\mathbf{F}$ be a splitting Jordan algebra. Then $\mathbf{F}_{+}$is splitting.

This is an easy application of proposition 1.

PROPOSITION 4. Two splitting Jordan subalgebra are canonically Jordan isomorphic.

Namely, let $\mathbf{F}$ and $\mathbf{F}^{\prime}$ denote two splitting Jordan subalgebra and $\mathbf{a} \in \mathbf{F}$. There is exactly one $\mathbf{a}^{\prime} \in \mathbf{F}^{\prime}$ such that $\mathbf{a}-\mathbf{a}^{\prime} \in \mathbf{S}$. This defines canonically a one-to-one mapping

$$
i_{F^{\prime}, \boldsymbol{F}}: \mathbf{a} \rightarrow \mathbf{a}^{\prime}
$$

from $\mathbf{F}$ onto $\mathbf{F}^{\prime}$. It is plain to see that this map is a Jordan isomorphism. Moreover, if both, $F$ and $F^{\prime}$, happen to be subalgebras then (6) is an isomorphism of algebras. Clearly,

$$
i_{F, F}=\text { id., } \quad i_{F, F^{\prime}} i_{F^{\prime}, F^{\prime \prime}}=i_{F, F^{\prime \prime}} .
$$

In a particular case we already know more about these morphisms:

$$
i_{F_{*}, F}=\left.P\right|_{F} \text {. }
$$

We now consider the morphism which is invers to (8). Let us write

$$
i_{F, F_{+}} \mathbf{a}=\mathbf{a}+r(\mathbf{a}), \quad \mathbf{a} \in \mathbf{F}_{+} .
$$

Let $\mathbf{a}, \mathbf{b}$ be two elements of the commutative algebra $\mathbf{F}_{+}$. Then

$$
2(\mathbf{a b}+r(\mathbf{a b}))=(\mathbf{a}+r(\mathbf{a}))(\mathbf{b}+r(\mathbf{b}))+(\mathbf{b}+r(\mathbf{b}))(\mathbf{a}+r(\mathbf{a})) .
$$

Taking into acount that $\mathbf{F}_{+}$is in the centre of $\mathbf{A}$ we get from this

$$
\forall \mathbf{a}, \mathrm{b} \in \mathbf{F}_{+}: r(\mathbf{a b})=r(\mathbf{a}) \mathbf{b}+\mathbf{a} r(\mathbf{b}) .
$$

Hence $r$ is a derivation from $F_{+}$into $A$ satisfying in addition

$$
\forall \mathbf{a} \in \mathbf{F}_{+}: r(\mathbf{a}) \in \mathbf{A}_{-} .
$$

Let us denote by

$$
\operatorname{Deriv}\left(\mathbf{F}_{+}, \mathbf{A}_{-}\right)
$$

the set of all maps, $r$, satisfying (10) and (11). It is again elementary to see that every $r$ fulfilling (10) and (11) defines a splitting Jordan subalgebra which is the set of all elements 


$$
\mathbf{a}+r(\mathbf{a}) \text { with } \mathbf{a} \in \mathbf{F}_{+} .
$$

This reduces the question for all Jordan splitting subalgebras to those splitting subalgebras which are central ones.

PROPOSITION 5. Let $\mathbf{F}_{+}$be a splitting subalgebra consisting of even elements only and $r \in \operatorname{Deriv}\left(\mathbf{F}_{+}, \mathbf{A}_{-}\right)$then

$$
\mathbf{F}=\left\{\mathbf{a}+r(\mathbf{a}), \mathbf{a} \in \mathbf{F}_{+}\right\}
$$

is a splitting Jordan subalgebra of $\mathbf{A}$ and every such algebra can be obtained by varying $\mathbf{F}_{+}$and $r$.

An odd derivation of $\mathbf{A}$ is by definition a linear map, $R$, of $\mathbf{A}$ into $\mathbf{A}$ satisfying

$$
w R+R w=0 \text { and }
$$

An odd derivation maps $\mathbf{A}_{+}$into $\mathbf{A}_{-}$.

LEMMA 6. Let $r \in \operatorname{Deriv}\left(\mathbf{F}_{+}, \mathbf{A}_{-}\right)$. If there is an odd derivation, $R$, of $\mathbf{A}$ the restriction of which to $\mathbf{F}_{+}$coincides with $r$ then

$$
T=\mathrm{id} .+R P
$$

is a Jordan automorphism of $\mathbf{A} . T$ maps $\mathbf{F}_{\mathrm{r}}$ onto $\mathbf{F}$ as defined by (14).

(17) coincides with $i_{F, F_{+}}$on $\mathrm{F}_{+} \cdot$ (15) shows

$$
\text { (id. }-w) R=R(\text { id. }+w) \text {, }
$$

and from this we infer

$$
T^{-1}=\text { id. }-R P \text {. }
$$

On the other hand we get by a short calculation

$$
\forall \mathbf{a} \in \mathbf{A}:(T \mathbf{a})^{2}=T\left(\mathbf{a}^{2}\right)
$$

showing $T$ is Jordan.

Lemma 6 reduces the problem to list all splitting Jordan subalgebras to the description of all even splitting subalgebras, provided we can obtain all elements of Deriv $\left(\mathbf{F}_{+}, \mathbf{A}_{-}\right)$by restricting suitable odd derivations.

From (18) or from (17) one obtains

$$
T w T w=\mathrm{id} . \quad \text { and } \quad w T w=T^{-1} .
$$


A map with this property we shall call $\ll w$-inverse».

\section{THE CARTAN ALGEBRA}

From now we assume $\mathbf{A}$ to be the Cartan algebra of smooth exterior differential forms on a given manifold,

$$
M, \quad \operatorname{dim} M=m .
$$

Every element $\mathbf{a} \in \mathbf{A}$ can be uniquely decomposed

$$
\mathbf{a}=\mathbf{a}_{(0)}+\mathbf{a}_{(1)}+\ldots+\mathbf{a}_{(m)}
$$

where $\mathbf{a}_{(0)}$ is a smooth function and $\mathbf{a}_{(k)}, k>0$, is an exterior differential form of class $C^{\infty}$. The radical, $\mathbf{S}$, of $\mathbf{A}$ consists of all elements $(22)$ with $\mathbf{a}_{(0)}=0$. One introduces in $\mathbf{A}$ a superstructur, $w$, by

$$
w(\mathbf{a})=\Sigma(-1)^{k} \mathbf{a}_{(k)} .
$$

The pair $\mathbf{A}, w$ is the supercommutative algebra we are aiming at, i.e. the Cartan algebra of $M$. Denoting by $\mathbf{A}_{(k)}$ the set of homogeneous elements of degree $k$, i.e. the set of all elements (22) with $\mathbf{a}_{(j)}=0$ for $j \neq k$, we see the direct sum decompositions

$$
\mathbf{A}_{+}=\mathbf{A}_{(0)}+\mathbf{A}_{(2)}+\ldots, \quad \mathbf{A}_{-}=\mathbf{A}_{(1)}+\mathbf{A}_{(3)}+\ldots
$$

and

$$
\mathbf{A}=\mathbf{A}_{(0)}+\mathbf{S}
$$

Thus. $\mathbf{A}_{(0)}$ is a splitting subalgebra containing only even elements. Let $\mathbf{F}$ be another splitting even subalgebra and let us consider the canonical isomorphism between them. There is an integer, $k$, with

$$
\mathbf{a} \in \mathbf{A}_{(0)}: \mathbf{a}-i_{F, A_{(0)}} \mathbf{a} \in \mathbf{S}^{2 k} .
$$

because both algebras contain even elements only. Hence we may write

$$
\forall \mathbf{a} \in \mathbf{A}_{(0)}: \mathbf{a}=i_{F, A_{(0)}} \mathbf{a}+q(\mathbf{a})+o(\mathbf{a})
$$

where $o(\mathrm{a})$ is contained in $\mathrm{S}^{2 k+2}$.

$$
\forall \mathbf{a} \rightarrow q(\mathbf{a}), \quad \mathbf{a} \in \mathbf{A}_{(\mathbf{0})},
$$

is a linear map from $\mathbf{A}_{(0)}$ into $\mathbf{A}_{(2 k)}$. Using the fact that $i_{F, A}$ is an ismorphism we get

$$
\forall \mathbf{a}, \mathbf{b} \in \mathbf{A}_{(0)}: q(\mathbf{a b})=q(\mathbf{a}) \mathbf{b}+\mathbf{a} q(\mathbf{b})
$$


Thus $q$ is a derivation from $\mathbf{A}_{(0)}$ into $\mathbf{A}_{(2 k)}$. Applying onto $q$ any sequence of $2 k$ pull backs with 1 -forms, we get a derivation from $\mathbf{A}_{(0)}$ into itself. But every such derivation is known to be a certain Lie derivation. Hence there exists a vector differential of degree $2 k$, denoted by $\vec{b}=\left\{\mathbf{b}^{j}\right\}$, such that locally on $M$

$$
\forall \mathbf{a} \in \mathbf{A}_{(0)}: q(\mathbf{a})=\mathbf{b}^{j}\left(\partial / \partial x^{j}\right) \mathbf{a} .
$$

Let now $\vec{E}=\left\{E_{j}\right\}$ denote the covector operator of pulling back the coordinate differentials, i.e.

$$
\left.E_{j} \mathbf{a}=\mathrm{d} x^{j}\right\lrcorner \mathbf{a}, \text { locally. }
$$

(The notation $《 E_{j} »$ for this operation is due to Kähler). Then the expression

$$
L:=\left(\mathbf{b}^{j} E_{j}\right) \mathrm{d}+\mathrm{d}\left(\mathbf{b}^{j} E_{j}\right)
$$

where «d»denotes total differentiation, is an even derivation of $\mathbf{A}$ into $\mathbf{A}$ that coincides on $\mathbf{A}_{(0)}$ with $q$. It is, indeed, a Nijenhuis derivation [7]. Let

$$
T_{2 k}:=\text { id. }+L+(1 / 2) L L+\ldots
$$

be the Lie series $\exp L$. It terminates for $L$ is nilpotent. As a consequence $T_{2 k}$ is an automorphism. It is

$$
T_{2 k} w=w T_{2 k}, \quad \mathrm{~d} T_{2 k}=T_{2 k} \mathrm{~d} .
$$

Now we consider the algebra

$$
\mathbf{F}^{\prime}=T_{2 k}^{-1}(\mathrm{~F})
$$

which is again an even splitting subalgebra. It satisfies

$$
\forall \mathbf{a} \in \mathbf{A}_{(0)}: \mathbf{a}-i_{F^{\prime}, A_{(0)}} \mathbf{a} \in \mathbf{S}^{2 k+2} .
$$

The radical $\mathbf{S}$ being nilpotent we can now conclude by induction

THEOREM 7. Let $\mathbf{F}$ ba a splitting subalgebra of A containing only even elements. Then there exists an automorphism, $T$, with

$$
\mathbf{F}=T\left(\mathbf{A}_{(0)}\right)
$$

and

$$
w T=T w, \quad \mathrm{~d} T=T \mathrm{~d}
$$

Indeed, we may choose for that purpose an automorphism, $T$, which is of the form $T_{2} T_{4} T_{6} \ldots$ where the $T_{2 k}$ are given by suitable automorphisms (33), (32).

Our next aim is a similar construction for splitting Jord an subalgebras. Taking 
into account lemma 6 we need to prove

LEMMA 8. Let $r \in \operatorname{Deriv}\left(\mathbf{F}_{+}, \mathbf{A}_{-}\right)$, where $\mathbf{F}_{+}$is a splitting even subalgebra. Then there is an odd derivation, $R$, of $\mathbf{A}$ coinciding on $\mathbf{F}_{+}$with $r$.

Proof. Using theorem 7 we may restrict ourself to the special case

$$
\mathbf{F}_{+}=\mathbf{A}_{(0)} \text {. }
$$

Further, Deriv $\left(\mathbf{A}_{(0)}, \mathbf{A}_{-}\right)$is the direct sum of the vector spaces Deriv $\left(\mathbf{A}_{(0)}, \mathbf{A}_{(2 k+1)}\right)$. Arguing, for every $k$, as in the case of the derivation (28), (29) and adding the resulting vector forms we see the existence of an odd vector differential, $\overrightarrow{\mathbf{b}}=\left\{\mathbf{b}^{j}\right\}$, with

$$
\forall \mathbf{a} \in \mathbf{A}_{(0)}: r(\mathbf{a})=\mathbf{b}^{j}\left(\partial / \partial x^{j}\right) \mathbf{a} .
$$

But for odd $\vec{b}$ the a la Nijenhuis defined operator

$$
R:=\left(\mathbf{b}^{j} E_{j}\right) \mathrm{d}-\mathrm{d}\left(\mathbf{b}^{j} E_{j}\right)
$$

turns out to be an odd derivation [7] the restriction on $\mathbf{A}_{(0)}$ of which coincides with $r$. Hence lemma 8 is valid if $\mathbf{F}_{+}=\mathbf{A}_{(0)}$. But the general assertion follows easily by the help of theorem 7 .

Now the corresponding Jordan automorphism

$$
T=\text { id. }+R P
$$

is the one to use in the situation described by lemma 6 .

THEOREM 9. Let F be a splitting Jordan subalgebra of A. Then there exists an even automorphism, $T_{1}$, and a w-inverse Jordan automorphism, $T_{2}$, such that

$$
\mathbf{F}=T\left(\mathbf{A}_{(0)}\right), \quad T=T_{2} T_{1} .
$$

Indeed, we first to to $F_{+}$by the described above Jordan automorphism. Then we use theorem 7 .

One finds the set of all products $T_{2} T_{1}$ where $T_{1}$ is even and $T_{2}$ is $w$-inverse ( - and constructed according to the procedures described above -) forming a group.

In this group the $w$-inverse Jordan automorphisms constitute a normal subgroup. The whole group is a semidirect product of that normal subgroup with the subgroup of its even elements.

In the case $m=\operatorname{dim} M$ is odd there are $w$-inverse Jordan automorphisms of the form (41) which are ordinary automorphisms. They are obtained by perform- 
ing the construction with vector differentials of degree $m$. Then these $w$-inverse automorphisms form a commutative subgroup of the normal subgroup of $w$ -inverse Jordan automorphisms.

\section{MANIFOLDS CANONICALLY ASSOCIATED TO THE CARTAN ALGEBRA}

The following construction can be performed for every smooth supermanifold which is given by their algebra of smooth superfunctions. The smoothness is necessary. In the continuous case the following construction becomes trivial.

We restrict ourseves, however, to the Cartan algebra, $\mathbf{A}$, of smooth exterior differentials defined on a manifold, $M$.

Let $m=\operatorname{dim} M$ and let $\Lambda$ be the unital Grassmann algebra over the reals generated by $m$ Grassmann variables.

DEFINITION. An ideal, $\mathbf{J}$, of $\mathbf{A}$ is called « $G$-point» iff $\mathbf{A} / \mathbf{J}$ is isomorphic to $\Lambda$.

A Jordan ideal, $\mathbf{J}$, of $\mathbf{A}$ is called « $J G$-point» iff $\mathbf{A} / \mathbf{J}$ is Jordan isomorphic to $\Lambda$.

Every $G$-point is at the same time a $J G$-point.

If $\mathbf{J}$ is a $J G$-point there is a maximal ideal, $\mathbf{I}$, of $\mathbf{A}$ with $\mathbf{A} / \mathbf{I}$ isomorphic to the real number field and $\mathbf{J} \subseteq \mathbf{I}$.

Indeed, assume $\beta$ to be a Jordan isomorphism of $\mathbf{A}$ onto $\Lambda$. Then every nilpotent element of $\mathbf{A}$ will be mapped onto a nilpotent element of $\Lambda$. Hence $\beta(\mathbf{S})$ is contained in the radical of $\Lambda$, and there is a proper Jordan ideal $\mathbf{I}$ in $\mathbf{A}$ containing $\mathbf{J}$ and $\mathbf{S}$. This Jordan ideal I induces a Jordan homomorphism from $\mathbf{A}$ onto the reals. Then $\mathbf{A}_{(0)} / \mathbf{I} \cap \mathbf{A}_{(0)}$ is Jordan isomorphic to the field of real numbers. Both algebras being commutative this is an ordinary isomorphism. Therefore there is a point $p \in M$ such that $\mathbf{I} \cap \mathbf{A}_{(0)}$ consists of all functions vanishing at $p$. But $\mathbf{I}$ is generated as a Jordan ideal by $I \cap \mathbf{A}_{(0)}$ and $S$. Taking into account that $\mathbf{A}_{(0)}$ is a splitting algebra one sees that $I$ is an ideal. It is plain that $\beta(I)$ is in the radical of $\Lambda$ for there is only one maximal ideal in the Grassmann algebra, its radical. Hence $\beta\left(\mathbf{I}^{m+1}\right)$ contains only the zero element. Thus we have proved

LEMMA 10. Let $\mathbf{J}$ be a $J G$-point. Then there is a point $p \in M$ such that the maximal ideal $\mathbf{I}_{p}$ of all elements the zero component of which vanishes at $p$ satisfies

$$
\mathbf{I}_{p}^{m+1} \subseteq \mathbf{J} \subseteq \mathbf{I}_{p}
$$

The set of all $J G$-point is, therefore, a subset of the so-called $m^{\text {th }}$ infinitesimal neighbouthood of the supermanifold the fibres of which are given by $\mathbf{A} / \mathbf{I}_{p}^{m+1}$ where $p$ runs through all the points of $M$. 
Our next aim is to show: The group of Jordan automorphisms introduced at the end of the last section acts transitive on the set of all $J G$-points. Let $\beta$ denote a Jordan homomorphism from $\mathbf{A}$ onto $\Lambda$, and let $e_{1}, e_{2}, \ldots, e_{m}$ be a Grassmann base of $\Lambda$

We may write

$$
\forall \mathbf{b} \in \mathbf{A}: \beta(\mathbf{b})=\beta_{0}(\mathbf{b})+\beta_{1}(\mathbf{b})+\beta_{2}(\mathbf{b})+\ldots
$$

with

$$
\beta_{j}(\mathbf{b})=\Sigma \beta_{j, i_{1}, \ldots, i_{j}}(\mathbf{b}) e_{i_{1}} \ldots e_{i_{j}}
$$

where all the coefficients in (45) are assumed to be real numbers. At first, $\mathbf{b} \rightarrow \beta_{0}(\mathbf{b})$ is a Jordan homomorphism onto the field of real numbers. It is $\beta_{0}(\mathbf{S})=$ $=0$ for there are no nilpotent real numbers. Thus $\beta_{0}$ induces an homomorphism of the commutative algebra $\mathbf{A}_{(0)}$ onto the real numbers. It follows the existence of a point, $p \in M$, with

$$
\forall \mathbf{g} \in \mathbf{A}_{(0)}: \beta_{0}(\mathbf{g})=\mathbf{g}(p) .
$$

Now assume $\beta_{1}=\ldots=\beta_{m}=0$, identically. Then the kernel $\mathbf{J}$ of $\beta$ is generated as an Jordan ideal by the ideal $\mathbf{I}_{p}^{0}$ of $\mathbf{A}_{(0)}$ consisting of all functions vanishing at $p$. This generating set is in the centre of the algebra. Therefore $\mathbf{J}$ is an ideal generated by $\mathrm{I}_{p}^{0}$, too. It consists of all differentials the coefficients of which vanish all at the point $p$ of $M$.

Next we assume the existence of an integer, $k$, such that for $1 \leqslant j<k$ it is $\beta_{j}=0$ identically, but $\beta_{k} \neq 0$.

Using the uniqueness of the decomposition (44) we conclude then

$$
\forall \mathbf{g}_{1}, \mathbf{g}_{2} \in \mathbf{A}_{(0)}: \beta_{k}\left(\mathbf{g}_{1} \mathbf{g}_{2}\right)=\mathbf{g}_{\mathbf{1}}(p) \beta_{k}\left(\mathbf{g}_{2}\right)+\mathbf{g}_{2}(p) \beta_{k}\left(\mathbf{g}_{1}\right)
$$

Performing any sequence of $k$ pull backs with the Grassmann generators $e_{1}, \ldots, e_{m}$ we arrive at a derivation from $\mathbf{A}_{(0)}$ into the real numbers. Taking into account (43) one concludes that these objects have to be Lie derivatives taken at the point $p$. Hence $\beta_{k}$ can be written in the following form: There is a (local) vector differential, $\overrightarrow{\mathbf{c}}$, of degree $k$ with

$$
\forall \mathbf{g} \in \mathbf{A}_{(0)}: \beta_{k}(\mathbf{g})=\left(\mathbf{c}^{i}\left(\partial / \partial x^{i}\right) \mathbf{g}\right)(p) .
$$

Depending whether $k$ is even or odd we choose an even or an odd derivation $L$ of $\mathbf{A}$ with the property

$$
\forall \mathbf{g} \in \mathbf{A}_{(0)}: \beta_{k}(\mathbf{g})=(L \mathbf{g})(p),
$$

which is possible in many ways using either (30), (32) if $k$ is even or, if $k$ is odd, (39), (40). 
Hence there is an automorphism (for even $k$ ) or a Jordan automorphism (for odd $k$ ), $T_{k}$, with the property

$$
\forall \mathbf{g} \in \mathbf{A}_{(0)}:\left(T_{k}^{-1} \beta\right)(\mathbf{g})=\mathbf{g}(p)+\beta_{k+1}^{\prime}(\mathbf{g})+\ldots .
$$

By induction we arrive at a Jordan automorphism, $T$, the property of which we describe by a theorem:

THEOREM 11. Let $\beta$ be a Jordan homomorphism from $\mathbf{A}$ onto $\Lambda$. Then there is a Jordan automorphism, $T$, of $\mathbf{A}$ and a point $p \in M$ such that

$$
\forall \mathbf{g} \in \mathbf{A}_{(0)}:\left(T^{-1} \beta\right)(\mathbf{g})=\mathbf{g}(p) .
$$

If $\beta$ is an homomorphism then $T$ can be chosen to be an automorphism of $\mathbf{A}$.

The last assertion arises this way: With the eventual exception of the highest degree, $\beta_{2 i+1}=0$ for homomorphisms.

We know already how the kernel of $\beta$ is constructed if the form (51) is reached. Taking advantage from the (Jordan) morphism properties we easely get:

THEOREM 12. Let $\mathbf{J}$ be a $J G$-point. Then there exists a point $p \in M$ and a splitting Jordan subalgebra, $\mathbf{F}$, such that $\mathbf{J}$ is Jordan generated by the intersection $\mathbf{F} \cap \mathbf{I}_{p}$. If $\mathbf{J}$ is a $G$-point then $\mathbf{F}$ can be chosen to be an algebra. The group of all automorphisms of $\mathbf{A}$ acts transitively on the set of all G-points. The group of the Jordan automorphisms acts transitively on the set of JG-points.

Indeed, the transitivity of the later groups if acting on those $G$-points resp. $J G$-points which belong to a fixed $p \in M$, i.e. $\mathbf{J} \subseteq \mathbf{I}_{p}$ is seen from the construction above. This transitivity is already true for the set of (Jordan) automorphisms constructed in section 3. The restriction to a fixed point of $M$ can be removed trivially for we have all the diffeomorphisms of $M$ which induce automorphisms of A: Let $S_{*}$ be a diffeomorphism of $M$. There is just one automorphism $S$ of $\mathbf{A}$ with

$$
\forall \mathbf{g} \in \mathbf{A}_{(0)}:(S \mathbf{g})(p)=\mathbf{g}\left(S_{*}\right), \quad \mathrm{d} S=S \mathrm{~d} .
$$

Let us denote by

$$
G \text {-space (A), } G_{+} \text {-space (A), } J G \text {-space (A), }
$$

the set of all $G$-points, even $G$-points, and $J G$-points, respectively. Here an even point is a point which is generated as an ideal by the intersection of a point ideal, $I_{p}$, and an even splitting subalgebra. It is not difficult to see that all sets (53) are closed subsets of the $m^{\text {th }}$ infinitesimal neighbourhood of $\mathbf{A}, m=\operatorname{dim} M$, i.e. are 
closed subsets of a smooth fibre bundle over $M$.

On the closed subsets (53) of that fibre bundle act large groups transitively. However, taking into account the constructions of section 3 we see that, locally, finite-dimensional subgroups are already sufficient to reach, from a given point, a whole neighbourhood. Therefore, the sets (53) turns out to be genuine smooth submanifolds of the $m^{\text {th }}$ infinitesimal neighbourhood. Furthermore, from this observation one can deduce the dimensionality of that manifolds. In doing so we get

THEOREM 13. There is a unique way to equipp the sets $G$-space (A), $G_{7}$-space (A), and JG-space (A) with the structure of a smooth manifold in such a manner that the group of all automorphisms, of all even automorphisms, and of all Jordan automorphisms of $\mathbf{A}$ acts respectively as a group of diffeomorphisms.

LEMM A 14. It is, under the assumption

$$
\begin{aligned}
& \operatorname{dim} M=m: \\
& \operatorname{dim} G \text {-space }(\mathbf{A})=m 2^{m-1} \quad \text { if } m \text { is even } \\
& \operatorname{dim} G \text {-space }(\mathbf{A})=m\left(2^{m-1}+1\right) \quad \text { if } m \text { is odd } \\
& \operatorname{dim} G_{+}-\operatorname{space}(\mathbf{A})=m 2^{m-1} \\
& \operatorname{dim} J G-\operatorname{space}(\mathbf{A})=m 2^{m} .
\end{aligned}
$$

Thus the dimensions of the space are, respectively,

$$
\begin{array}{ll}
\text { For } m=1: 2,1,2 . & \text { For } m=2: 4,4,8 . \\
\text { For } m=3: 15,12,24 . & \text { For } m=4: 32,32,64 .
\end{array}
$$

The superstructure $w$ is represented as a reflection of $J G$-space on its subspace $G_{+}$-space.

\section{REFERENCES}

[1] F.A. BEREZIN, Introduction to Algebra and Analysis with anticommuting Variables, Edit. Moscow University, Moscow, 1983. (in Russian).

[2] B. Kostant, Graded Manifolds, graded Lie Theory, and Prequantisation, Lecture Notes in Math., 570, Springer-Verl., Heidelberg, N.Y., 1977.

[3] D.A. LEITES, Introduction to the Theory of Supermanifolds, Uspechi mat. nauk., 35 (1980) 3 - 57. (in Russian).

[4] Th. SCHмIT, Elements of Super Differential Geometry, IMATh preprint, Berlin, 1984.

[5] A. UhlmanN, On Associative Algebras of Superfunctions, in "Quantum Theory of Particles and Fields», World Scientific Publ., Singapore, 1983, 188 - 200. 
[6] A. Uhlmann, Die Cartan-Algebra der Ausseren Differentialformen als Supermannigfaltigkeit: Automorphismen, Jordan-Automorphismen und ihre Realisierung als Diffeomorphismen, ZiF preprint 57, Bielefeld 1984, $1-21$.

[7] A. Trautmann, The Lie Algebra of the Cartan Algebra, Lecture at the Banach Centre, Warshawa 1983, to be published.

Manuscript received: June 19, 1984. 